\title{
La relevancia de la investigación durante la formación de pregrado
}

Ana Carolina Arévalo ${ }^{1}$

Ligia Medina ${ }^{2}$

\section{$\mathrm{PC} /$ Estuvieron a cargo de guiar un proyecto que culminó en la revista de divulgación científica Fitofarma. ¿Cómo plantearon el proyecto para trabajar con los estudiantes de la asignatura de Farmacognosia?}

AA/ Ambas impartimos la clase de Farmacognosia I, que se relaciona con el estudio de productos naturales; en particular, el enfoque principal de la clase es el estudio de plantas y lo orientamos a plantas medicinales por el área de química y farmacia. Nuestro objetivo principal es involucrar a los estudiantes en procesos de investigación, así que queremos que nuestra clase sea un ente de cambio, que no solamente sea para darles el conocimiento científico, sino que tengan una actitud diferente, que sepan que existe un mundo totalmente desconocido, y que es necesario que nosotros nos interesemos en él y a través de la investigación lo podemos hacer. El objetivo principal es que nuestros estudiantes aprendan 0 lleven un proceso de investigación y cuando empezamos a organizar cuáles eran las ventajas y los recursos que teníamos se nos ocurrió plantear el proyecto, donde los estudiantes empiezan a llevar esos conocimientos que vemos tanto en teoría como en práctica a una vinculación a las diferentes comunidades. Nosotros estructuramos el proyecto, en una forma de acompañamiento a los estudiantes y porque debía ser un proceso bien estructurado y guiado, y luego lo compartimos con ellos. Lo primero que se hace es el contacto con las comunidades a través de encuestas etnobotánicas, una vez que tenemos las encuestas etnobotánicas, escogemos cuál va a ser la planta de estudio en base a criterios de novedad, es decir que no haya sido muy estudiada y que no tenga muchos reportes, porque si no, no tendría el fin principal que es generar conocimiento nuevo.

\footnotetext{
${ }^{1}$ Profesora investigadora de la Facultad de Química y Farmacia, Doctora en Ciencias Farmacéuticas Universitá Degli Studi di Salermo, Italia. Doctora en Ciencias Químicas y Farmacia en el grado de licenciatura, UNAH. Miembro activo del grupo de investigación "Productos Naturales", adscrito a la Facultad de Química y Farmacia, UNAH: ana.arevalo@unah.edu.hn

${ }^{2}$ Profesora investigadora de la Facultad de Química y Farmacia. Magister en Quimica, Universidad de Costa Rica. Doctora en Ciencias Químicas y Farmacia en el grado de licenciatura, UNAH. Miembro activo del grupo de investigación "Productos Naturales", adscrito a la Facultad de Química y Farmacia, UNAH: liamel94@yahoo.com
} 
Una vez que tenemos las encuestas etnobotánicas, escogemos la planta por origen de los estudiantes, especialmente si son de determinados departamentos; eso genera mayor identidad y responsabilidad con el proyecto. Una vez que ya se tienen las encuestas etnobotánicas se empiezan a desarrollar los estudios en laboratorio.

LM/ Aparte de la clase nosotros involucramos lo que es el grupo de investigación en productos naturales, en el cual tenemos estudiantes. Como decía la doctora Carolina Arévalo, queremos que los estudiantes pasen por todo el proceso, desde hacer una encuesta etnobotánica y llevarla al campo, desarrollar una parte en el laboratorio, y luego culminar con una publicación. Tenemos tanta cantidad de información recopilada por los

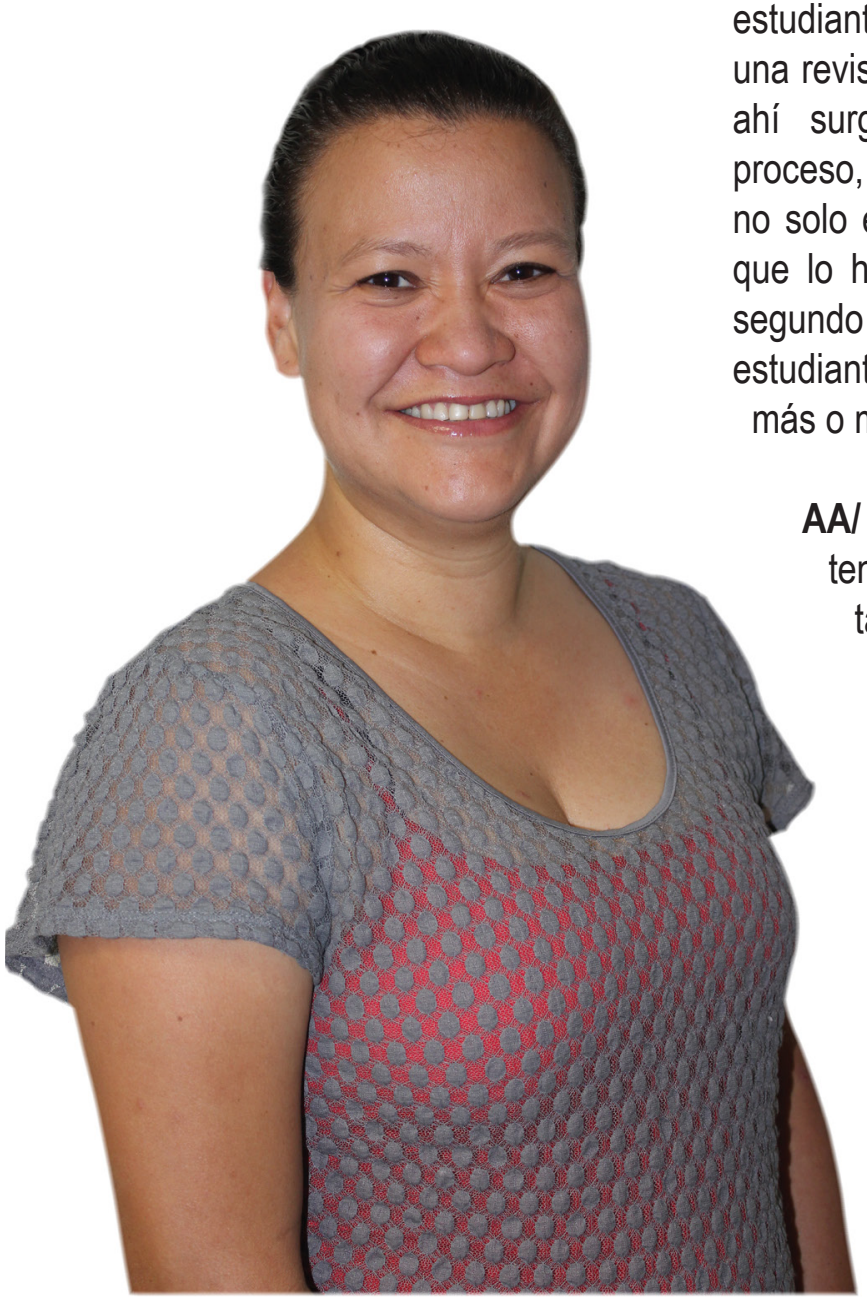

Ligia Medina
AA/ Por la responsabilidad que tenemos de realizar una revista de divulgación era muy importante que los resultados fueran validados antes de colocarlos en la revista. Por eso es que se planteó un proyecto cuya duración tenía un año, para que tuviéramos las diferentes etapas de validación. Nos pareció importantísimo que nosotros tuviésemos esta revista porque es la forma de devolver el 
conocimiento validado a las diferentes comunidades; ya que extrajimos la información que ha pasado de generación en generación y a través de los estudios que se realizaron en el laboratorio validamos ese conocimiento aplicando una metodología científica.

\section{$\mathrm{PC} /$ En cuanto a la enseñanza de la investigación, ¿qué buenas prácticas han encontrado para que ellos asimilen el proceso que esta conlleva?}

AA/ Lo mejor es involucrar a los estudiantes desde el inicio, comentamos el trabajo o proceso de investigación de Farmacognosia I, cómo será llevado a lo largo de todo el período para culminar con una publicación científica, y eso genera responsabilidad en ellos, y un cambio de actitud que pudimos observar en los chicos que participaron. Tuvimos el lanzamiento de la revista, que era la culminación de todo el proyecto, y fueron los estudiantes los encargados de organizarse en la logística, hacer las presentaciones de las plantas a las personas que asistieron, y eso generó una apropiación del trabajo: "es el fruto de mi trabajo de investigación". Realmente se apropiaron de ese conocimiento y cambiaron de actitud. Creo que nuestros procesos de investigación se llevan de manera transversal con el conocimiento teórico y con el conocimiento práctico en los diferentes laboratorios. Eso es importante porque no podemos ser profesores si no hacemos investigación y si queremos lograr un cambio de actitud, tiene que ser a través de este tipo de procesos.

LM/ En primer lugar, el acompañamiento del estudiante, como la doctora Carolina Arévalo mencionó en alguna presentación que hizo, trabajamos de manera artesanal. Nosotros vamos con los estudiantes en todo el proceso, incluyendo tanto en la clase que es la teoría como en el laboratorio, con la Dra. Laura, que está en el laboratorio. Tenemos esa unión de la clase con el laboratorio muy sólida, que es una buena práctica porque esa unión la llevamos de la mano. La importancia del acompañamiento del estudiante desde que hace la encuesta, revisarla, también con la tabulación de datos, estar pendiente del laboratorio, revisar los resultados, hasta lo que es la redacción de la publicación. Todo eso es muy importante, estar con el alumno y no solo que sienta que es una asignación sin contar con el docente.

AA/ Hemos tenido bastante experiencia con el grupo de investigación, ya que tenemos bastantes estudiantes dentro del grupo, que son personas que entran y salen, así que hemos logrado generar un esquema de trabajo que también es aplicable a nuestra clase. En cada etapa de nuestros proyectos de investigación 
hemos identificado cuáles son los objetivos de aprendizaje, algo que hemos logrado con la experiencia en el grupo de investigación.

$\mathrm{PC} / \mathrm{Si}$ se aseguran de enseñar la investigación, ¿cómo evalúan el aprendizaje de los estudiantes con relación a la investigación?

AA/ Hemos tenido una respuesta muy buena de parte de los estudiantes, creo que el hecho de estar siempre concientizando que lo que ellos están haciendo es importante, que tendrá un impacto en el nivel de la sociedad es una de las herramientas que más utilizamos. También lo señala la doctora Ligia Medina, el acompañamiento.

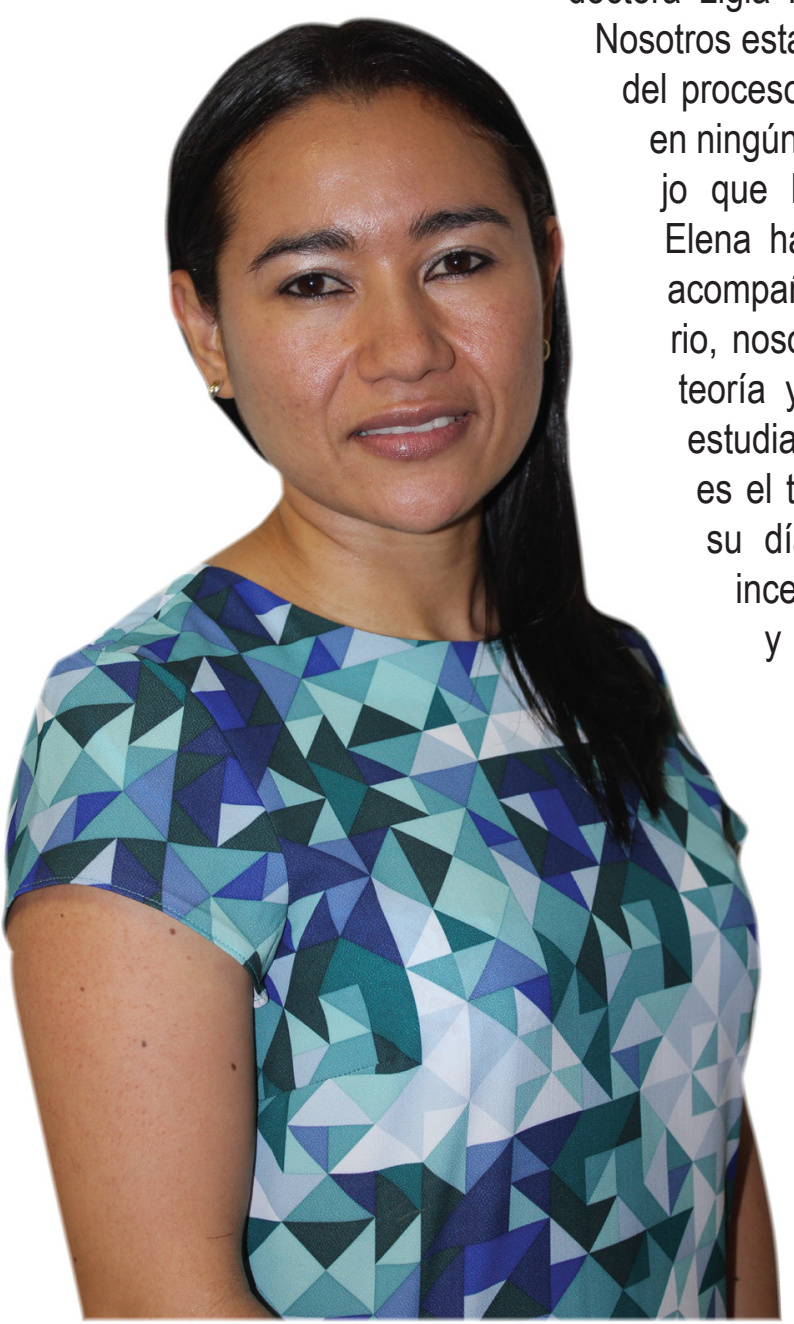

Ana Carolina Arévalo osotros estamos con ellos en cada etapa el proceso, no los dejamos a la deriva en ningún momento, entonces el traba- que ha hecho la doctora Laura Elena ha sido básico en relación al acompañamiento dentro del laborato, nosotros hacemos la parte de la eoría y la fe que tenemos en los estudiantes también abona, porque es el tenemos. Estar con ellos, ver su día a día, tratar con ellos e incentivar los cambios de actitud y la respuesta es buena, ver cómo se motivan y encontrar que la mayoría está muy interesado. De hecho, de todas estas experiencias se nutre el grupo de investigación y muchos quieren entrar, pero no siempre tenemos espacio para poder aceptarlos a todos.

LM/ La ventaja que tenemos es que vemos al estudiante a diario, entonces el acompaña- 
miento puede ser diario,

porque después de la clase hay preguntas o en el laboratorio las hacen, se pueden hacer sesiones de trabajo en un cronograma por días y hacer las revisiones de lo que están haciendo. También tenemos protocolos establecidos en los proyectos, por lo que se explica el protocolo que se seguirá, sobre todo en el laboratorio.

\section{Ilustración 1. Proceso de formación desarrollado por el Grupo de Investigación en Productos Naturales de la UNAH. (Cortesía de la coordina- ción del grupo)}

\section{PROCESO DE FORMACIÓN}

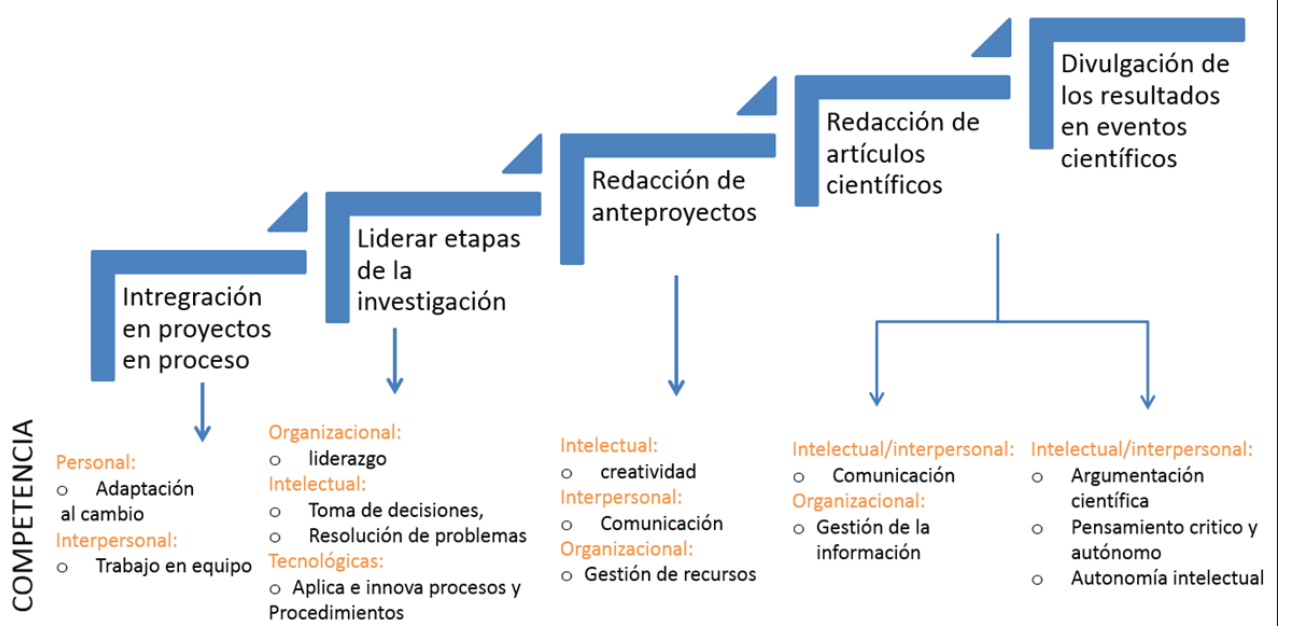

PC/ En cuanto a la ejecución de la revista, ¿cómo hicieron el trabajo de gestión y coordinación?

LM/ Trabajamos por grupos de laboratorio, entonces cada grupo tenía asignado elaborar una página de la revista que corresponde a una planta. Cada grupo tenía un coordinador, así que las reuniones de trabajo eran con los coordinadores, algo organizado principalmente por la doctora Laura Elena. Nos reuníamos con ella a revisar los trabajos y luego revisábamos cada página de la revista. Además, como grupo de investigación gestionamos recursos para poder publicarla. 
AA/ Una de las cosas que necesitábamos era que nuestra revista pudiera aportar a las personas de las comunidades, así que teníamos que idear una forma de que fuese atractiva visualmente y que el lenguaje no fuese muy técnico, porque el impacto es hacia estas personas que nos habían dado la información y a las cuales se la regresábamos. Se nos ocurrió realizar infogramas, cuya estructura definimos en consenso con los diferentes coordinadores de los grupos de trabajo y escogimos cuál era la información que incluiríamos en ellos. Luego vino el proceso de edición, desde darle un nombre a la revista, que fue discutido con los estudiantes, y así empezamos a estructurar la información que cada grupo tenía que aportar a los infogramas. Escogimos 24 plantas de alrededor de 200 de las que tenemos información, porque era un número manejable en ese momento; los recursos fueron en parte de los estudiantes y de nosotras, pero gracias a una propuesta enmarcada en las estrategias de fortalecimiento del grupo de investigación conseguimos financiamiento con la DICYP para un tiraje de 300 revistas.

\section{$\mathrm{PCl}$ ¿Cómo ha sido la planificación del trabajo de entregar esta revista de divul- gación y el conocimiento contenido en ella a las comunidades?}

AA/ Esa es la siguiente etapa, en la que convocaremos a todos los estudiantes que participaron, quizá algunos estén graduados, para que ellos nos acompañen a las comunidades a devolver la información. Pensamos enfocarnos en centros de salud y también en escuelas y alcaldías, que ellos tengan esta información y puedan utilizarla para organizar procesos de capacitación o que realmente la utilicen para fines de salud y educación.

LM/ Tenemos la idea de lograr la concienciación del correcto uso de plantas medicinales, que es una de las formas en que queremos devolver la información a la comunidad, no solo con la revista, sino también queremos lograr las capacitaciones que serían realizadas por los estudiantes y que esto se integra a su formación, el poder desenvolverse en comunicaciones orales, tiene todo un proceso desde que inicia en el laboratorio, llevar a cabo la comunicación escrita y luego la oral.

AA/ Otro propósito de la revista es hacer una base de datos con el conocimiento tradicional, porque es importante que este conocimiento no se pierda. Sabemos que se transmite de generación en generación, y si no hay un registro, los jóvenes pueden estar menos interesados ya que los informantes de este proyecto, de acuerdo a las estadísticas, fueron en su mayoría amas de casa o personas mayores de los 60 años. El hecho de tener un registro y de preservar ese conoci- 
miento tradicional es parte de nuestra identidad como pueblo.

$\mathrm{PCl}$ ¿Cómo pueden aportar los estudiantes, a través de la investigación, en estos procesos de clase?

LM/ Aportan desde una idea, ya que hay jóvenes que hemos tenido en clases y llegan a los grupos con ideas, que es, por ejemplo, el caso de la Beca Sustantiva que desarrollamos con la idea de un estudiante que se acercó y quería participar. Esto es una alegría, porque se vio la motivación y luego su incorporación; pero también se nota en la integración de un grupo que lleva todo un proceso complejo.

AA/ Como docentes no debemos olvidar que tenemos recurso en el aula y que tenemos la obligación de motivar y generar proyectos en los que ellos se puedan involucrar en todo el proceso, en cada etapa del trabajo, y recordar que también tenemos esa responsabilidad de motivarlos y enseñarles que el mundo es mucho más grande de lo que parece y que esa curiosidad es importante motivarla. 